\title{
Noise-immune image blur detection via sequency spectrum truncation
}

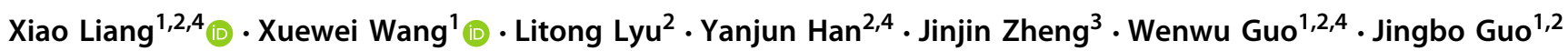

Received: 23 July 2021 / Accepted: 11 November 2021 / Published online: 9 December 2021

(C) The Author(s) 2021

\begin{abstract}
Blur detection is aimed to differentiate the blurry and sharp regions from a given image. This task has attracted much attention in recent years due to its importance in computer vision with the integration of image processing and artificial intelligence. However, blur detection still suffers from problems such as the oversensitivity to image noise and the difficulty in cost-benefit balance. To deal with these issues, we propose an accurate and efficient blur detection method, which is concise in architecture and robust against noise. First, we develop a sequency spectrum-based blur metric to estimate the blurriness of each pixel by integrating a re-blur scheme and the Walsh transform. Meanwhile, to eliminate the noise interference, we propose an adaptive sequency spectrum truncation strategy by which we can obtain an accurate blur map even in noise-polluted cases. Finally, a multi-scale fusion segmentation framework is designed to extract the blur region based on the clustering-guided region growth. Experimental results on benchmark datasets demonstrate that the proposed method achieves state-of-the-art performance and the best balance between cost and benefit. It offers an average $F_{1}$ score of 0.887 , MAE of 0.101 , detecting time of $0.7 \mathrm{~s}$, and training time of $0.5 \mathrm{~s}$. Especially for noise-polluted blurry images, the proposed method achieves the $F_{1}$ score of 0.887 and MAE of 0.101, which significantly surpasses other competitive approaches. Our method yields a cost-benefit advantage and noise immunity that has great application prospect in complex sensing environment.
\end{abstract}

Keywords Blur detection $\cdot$ Noise immunity $\cdot$ Sequency spectrum $\cdot$ Walsh transform

\section{Introduction}

In recent years, many vision-related issues have been evaluated with the integration of image processing and artificial intelligence. Blur is an undesirable but widespread visual phenomenon that heavily degrades the quality of the captured image from a sensing system. Many factors like motion and

Xuewei Wang

xwwang@stdu.edu.cn

$凶$ Jingbo Guo

guojingbo66@163.com

1 State Key Laboratory of Mechanical Behavior and System Safety of Traffic Engineering Structures, Shijiazhuang Tiedao University, Shijiazhuang 050043, China

2 School of Mechanical Engineering, Shijiazhuang Tiedao University, Shijiazhuang 050043, China

3 Department of Precision Machinery and Precision Instrumentation, University of Science and Technology of China, Hefei 230026, China

4 Hebei Advanced Manufacturing Technology Innovation Center for Concrete Components, Shijiazhuang 050043 , China defocus can cause partial blur to images, as shown in Fig. 1. Blur detection is aimed to differentiate the blurry and sharp regions from a given image, which is a key task in many computer vision applications including but not limited to image restoration [1], image quality assessment [2], target detection [3], depth estimation [4], and image retargeting [5]. However, the image blur is usually with uneven distribution and indistinct boundary [6], and also easily mingled with lots of noises in realistic sensing and transporting processes [7]. These situations bring large difficulty to accurate blur detection.

In the past decade, many blur detection techniques have been proposed and classified roughly into unsupervised methods $[6,10,11,15-22]$ and supervised methods $[9,12$, 13, 24-30, 38-40]. Both methods have made remarkable contributions to this field. The unsupervised class generally has explicit mechanism, good interpretability, and low complexity, but also has the difficulty of balancing accuracy and efficiency [8]. The supervised class is usually data driven and has a high performance, but requires considerable manually annotated samples, which are often unavailable in realistic detection tasks. More importantly, most existing blur detection methods are susceptible to image noise 


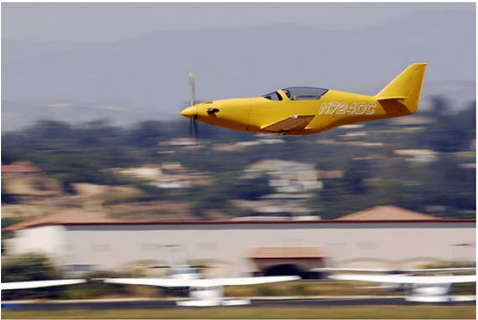

(a)

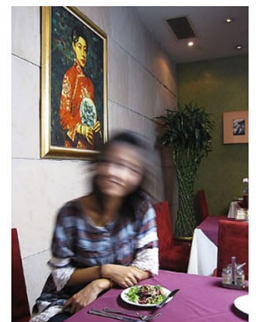

(b)

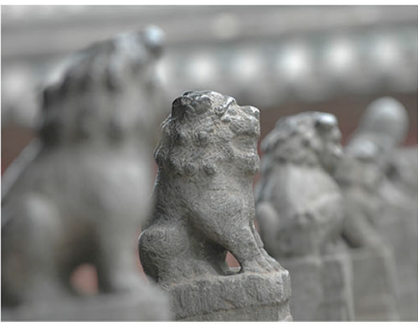

(c)

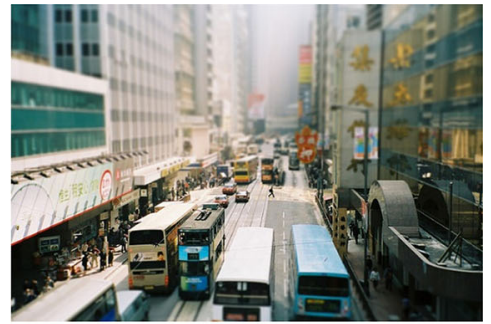

(d)

Fig. 1 Some partially blurred images. a, b Are motion-blurred cases. c, $\mathbf{d}$ Are defocus-blurred cases

so that their performance is greatly limited when faced with noise-polluted cases.

To address these problems, we propose a noise-immune blur detection method based on sequency spectrum truncation. The uniqueness of our method is summarized as follows. (i) A fast pixel-wise blur metric is constructed in sequency domain based on the Walsh transform. By virtue of the Walsh basis function containing only +1 and -1 , which can be easily compiled by computer, our method has a quite low computing cost. (ii) A noise-immune blur detection framework is proposed via adaptively truncating the sequency spectrum, based on the observation that noises preferentially occupy the high-sequency zones while blurry pixels are associated with low sequencies. (iii) Overall, the proposed method achieves a superior performance and simultaneously a good time efficiency. Especially in noise-polluted cases, our method remarkably outperforms state-of-the-art approaches.

\section{Related works}

Currently, researches on blur detection can be mainly classified into two categories: the unsupervised methods based on frequency/gradient analysis and the supervised methods based on learning.

\section{Unsupervised methods}

The unsupervised blur detection method is usually based on a clear mechanism. The most frequently used mechanism is that the sharp region has larger gradient magnitude or more high-frequency components than the blur region. Lee et al. [11] developed a technique to divide the input image into nonblur, defocus blur, and motion blur regions based on gradient amplitude and directional coherence. Tang et al. [15] utilized the log averaged spectrum residual to get a coarse blur map and improved the result from coarse to fine based on gradient and color similarity of neighbor pixels. Zhang et al. [16] estimated the blur map by exploiting the edge information and K-nearest neighbors matting interpolation. Yi et al. [17] proposed a fast blur detection framework integrating local binary pattern, image matting and multi-scale inference. Xu et al. [19] used the maximum rank of the local image patch in gradient domain to estimate the spatially varying defocus blur. In [22], Liu et al. united region-based frequency information and edge-based linear information to estimate the defocus blur map via regression tree fields.

Some blur detection methods with other mechanisms are also effective. Su et al. [10] distinguished the blur region by combining pixel-wise singular values and alpha channel information. Golestaneh et al. [6] identified the blur region by multi-scale fusion, sorting and regularization of high-order DCT coefficients in gradient domain. In a previous work [20], we employed a re-blur scheme and DCT coefficients to detect the image blur. Javaran et al. [18] developed another DCTbased blur metric using a similar workflow with ours. Xiao et al. [21] detected the defocus blur based on the decomposition and fusion of multi-scale singular values.

\section{Supervised methods}

The supervised blur detection method is usually data driven and dependent on training the classifier. Liu et al. [9] trained a Bayesian bi-classifier based on the gradient, spectrum and color information to identify the blur region. Shi et al. combined the gradient distribution, Fourier frequencies and local filter to distinguish the blur region using the naive Bayesian classifier in [12] and detected the just noticeable defocus blur by sparse edge representation and blurriness estimation in [13].

Recently, the methods based on deep learning have attracted much attention. Park et al. [24] designed the convolutional neural network-based blur feature extractor and fully connected neural network-based classifier to detect the defocus blur. Huang et al. [25] built a six-layer network to predict pixel-wise blur probability and fused multi-scale blur probability maps into a fine result. Zhao et al. [26, 30] proposed a defocus blur detection method based on a fully convolutional network with multi-stream input and bottomtop-bottom framework. In [27], Zeng et al. developed a local blur metric composed of deep feature learning and principal component analysis at the super-pixel level. In another previ- 
ous work [28], we also built a deep encoder-decoder network equipped with multi-inputs and multi-losses to realize the end-to-end blur detection. Tang et al. [29] proposed a fully convolutional neural network named DeFusionNet, which recurrently fused and refined multi-scale deep features for defocus blur detection. Furthermore, the DeFusionNet got improved by embedding a feature adaptation module and a channel attention module to better exploit discriminative multi-scale features [38]. Tang and his team [39] also developed a deep network for efficient and accurate defocus blur detection via recurrently refining multi-layer residual features. Li et al. [40] designed a dual-branch attention-based network, which was capable of exploiting the complementary information between in-focus and out-of-focus pixels and combining the high-level and low-level features.

\section{Summary}

Those above-mentioned studies have made remarkable contributions to the field of blur detection in the past decade. Unsupervised methods are interpretable and feasible in mechanism, with low complexity. Deep learning-based methods can automatically extract suitable features to distinguish blurry pixels from an image, and usually have a high performance driven by a considerable amount of data. However, there are still some limitations: (i) most methods are difficult to achieve a cost-benefit balance; in other words, improving the detection performance is often at the expense of time efficiency; (ii) the deep learning-based methods need numerous annotated samples and sufficient computation power for training and inferring, which are laborious and expensive; (iii) most existing blur detection algorithms are oversensitive to image noise. It leads to quite a large limitation to their performance when facing with noised cases. Note that there are some techniques can help to mitigate the noise sensitivity, such as pre-denoising and anti-noise training, but these palliative techniques introduce extra costs. Therefore, to address these issues, we propose a fast but accurate blur detection method based on sequency spectrum truncation in this paper. It performs well for both noise-free and noisepolluted cases.

\section{Proposed algorithm}

The framework of the proposed method is shown in Fig. 2. First, the input image is artificially re-blurred using a kernel function. It can be observed that, after the re-blur process, the sharp region loses numerous details whereas the loss of detail for the blur region is far less. The re-blur scheme has been proved effective in inspecting the unknown blur in our previous work [20] and other literature [18]. Second, the pixel-wise blurriness (i.e., blur map) is measured by quantifying the difference between the input image and its reblurred version in the sequency domain which is derived by the Walsh transform. Here, an adaptive sequency spectrum truncation strategy is used to eliminate the noise interference so that we can handle the noise-polluted cases. Finally, a multi-scale fusion segmentation framework is designed to extract the accurate blur region based on clustering-guided region growth.

\section{Blur metric based on Walsh transform}

According to Harmuth's sequency theory [14], the sequency is defined as half of the average zero-crossing times per second for a function. Compared with the frequency, the sequency has a more generalized concept capable of rep-

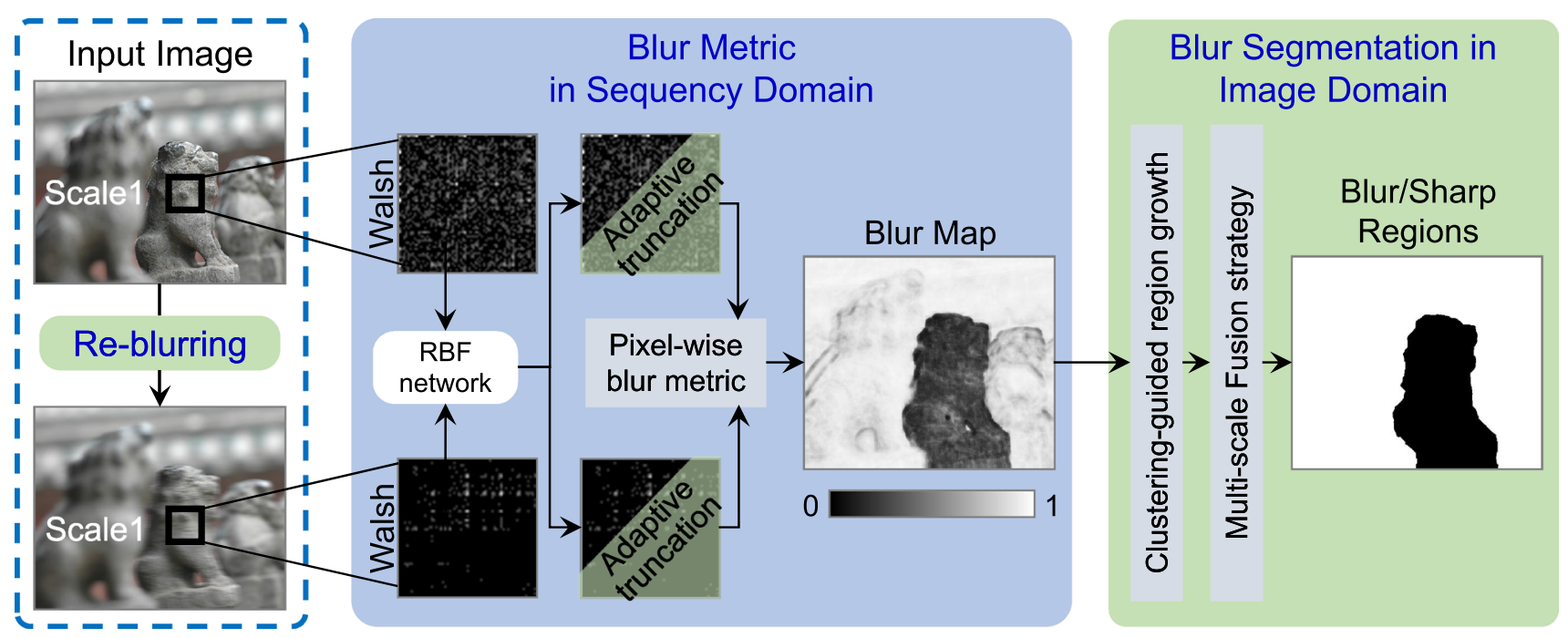

Fig. 2 The framework of the proposed blur detection method 
resenting both periodic and nonperiodic functions. In this paper, we convert the input image into sequency domain using the Walsh transform (one of orthogonal transforms) and hereby analyze the loss of image detail during the re-blur process. Unlike the Fourier transform and Cosine transform, the basis of Walsh transform is not the sinusoidal function but rather the Walsh function. It contains only two values of +1 and -1 in a certain sequence [35], and hence can be easily compiled by computer binary code; in other words, the Walsh transform has a quite low computing cost. For a patch $\boldsymbol{w} \in \mathbb{R}^{N \times N}$ in the input image $\boldsymbol{I} \in \mathbb{R}^{M \times M}$, the discrete Walsh transform is:

$\mathrm{W}_{h}(\mathrm{w})=\mathrm{W}_{h}(u, v)=\frac{1}{N^{2}} \sum_{x=0}^{N-1} \sum_{y=0}^{N-1} \mathrm{w}(x, y)\left[(-1)^{\phi(x, y, u, v)}\right]$,

where $\boldsymbol{W}_{h} \in \mathbb{R}^{N+N}$ is the sequency spectrum, $(x, y)$ denotes the pixel in $\boldsymbol{w},(u, v)$ denotes the element in $\boldsymbol{W}_{h}$. ($1)^{\phi(x, y, u, v)}$ is the Walsh basis function whose sequence of +1 and -1 is determined by $\phi(x, y, u, v)$. Here, we use a Hadamard sequence:

$\phi(x, u, v, y)=\sum_{i=0}^{n-1}\left[b_{i}(x) b_{i}(u)\right]+\sum_{j=0}^{n-1}\left[b_{j}(y) b_{j}(v)\right]$,

where $b_{i}(x)$ is the $i$ th value (either 0 or 1 ) of the reverse natural binary code of $x, N=2^{n}$. Therewith, the Hadamard sequence can be expressed in a recursive matrix mode starting from order 2 to order $n$ :

$\left[H_{2}\right]=\frac{1}{\sqrt{2}}\left[\begin{array}{cc}1 & 1 \\ 1 & -1\end{array}\right],\left[H_{h}(n)\right]=\left[H_{2}\right] \ominus\left[H_{h}(n-1)\right]$,

where $\odot$ denotes the Kronecker product. Consequently, the Eq. (1) can be expressed as:

$\mathrm{W}_{h}(\mathrm{w})=\left[H_{h}(n)\right] \times \mathrm{w} \times\left[H_{h}(n)\right]^{T}$.

Consistent with other orthogonal transforms, the non-zero $\boldsymbol{W}_{h}$ coefficients in sequency spectrum can represent image information. Considering that the loss of non-zero coefficients during the re-blur process in a clear region is far more than that in a blur region, we can utilize the loss to estimate the blur level. Therefore, we design a norm descriptor $\left\|\boldsymbol{W}_{h}\right\|_{L 2} \times$ $\left\|\boldsymbol{W}_{h}\right\|_{L \infty}$, in which the $L_{2}$ norm and the $L_{\infty}$ norm characterize the global cumulant and the local maximum of non-zero coefficients in $\boldsymbol{W}_{h}$, respectively. The integrated norm descriptor $\left\|\boldsymbol{W}_{h}\right\|_{L 2} \times\left\|\boldsymbol{W}_{h}\right\|_{L \infty}$ considering both global and local effects can be used to measure the quantity of non-zero coefficients in sequency spectrum. Therefore, we define the blurriness of one pixel in the input image $\boldsymbol{I}$ as follows:

$\lambda(\mathrm{w})=\frac{\left\|\mathrm{W}_{h}\left(\mathrm{w}^{b}\right)\right\|_{L_{2}} \times\left\|\mathrm{W}_{h}\left(\mathrm{w}^{b}\right)\right\|_{L_{\infty}}}{\left\|\mathrm{W}_{h}(\mathrm{w})\right\|_{L_{2}} \times\left\|\mathrm{W}_{h}(\mathrm{w})\right\|_{L_{\infty}}}$,

where $\boldsymbol{w} \in \mathbb{R}^{N \times N}$ and $\boldsymbol{w}^{b} \in \mathbb{R}^{N \times N}$ denote the pixel-centered patches before and after the re-blur process, respectively. Evidently, the blurriness $(\lambda)$ estimated using Eq. (5) is a value within $[0,1]$. The closer this value to 1 , the blurrier the pixel is. After estimating the blurriness of all pixels, we can obtain a blur map $\boldsymbol{\Lambda}$ for the entire input image. As shown in Fig. 3, the blur region has $\lambda=0.63-0.91$ and the sharp region has $\lambda=0.12-0.18$ using the re-blur kernel of a $9 \times 9$ mean filter. Visibly, the estimated blur map $\boldsymbol{\Lambda}$ can properly indicate the blur level of each pixel.

\section{Noise-robust blur metric via sequency spectrum truncation}

It is important to note that, if the input image is polluted by noise, the proposed blur metric in Eq. (5) will produce a quite lower value, which may result in a notable blur estimation error. Figure 4 shows such a case that the estimated $\lambda$ $=0.183$ for the noise-polluted blurry image is much smaller than the estimated $\lambda=0.749$ for the corresponding noise-free blurry image; in this case, the noise-polluted blurry image would be misjudged into a clear one. The reason is that those noise components in the blurry image bring in many high-sequency $\boldsymbol{W}_{h}$ coefficients and accordingly increase the norm descriptor $\left\|\boldsymbol{W}_{h}\right\|_{L 2} \times\left\|\boldsymbol{W}_{h}\right\|_{L \infty}$. Once the noise-polluted blurry image is re-blurred, its high-sequency $\boldsymbol{W}_{h}$ coefficients and corresponding norm descriptor will be reduced significantly. Therefore, the value calculated by Eq. (5) will be near to zero, which incorrectly indicates that the image is clear.

To address the estimation error in noisy conditions, we propose a sequency spectrum truncation strategy. It is assumed that image noise acquired by optical sensors is usually additive Gaussian white noise. Since the blur information is compressed to low-sequency spectrum zones while the noise preferentially concentrates in high-sequency spectrum zones, the noise effect can be reduced by truncating and discarding these high-sequency $\boldsymbol{W}_{h}$ coefficients. Hence, the modified blur metric is defined as:

$\lambda^{l}(\mathrm{w})=\frac{\left\|\mathrm{W}_{h}\left(\mathrm{w}^{b}\right)\right\|_{L_{2}}^{l} \times\left\|\mathrm{W}_{h}\left(\mathrm{w}^{b}\right)\right\|_{L_{\infty}}^{l}}{\left\|\mathrm{~W}_{h}(\mathrm{w})\right\|_{L_{2}}^{l} \times\left\|\mathrm{W}_{h}(\mathrm{w})\right\|_{L_{\infty}}^{l}}$,

where $l=\alpha N$ denotes the truncation length $(2 \leq l \leq N)$ in the sequency spectrum and $\alpha$ is the truncation proportion $(0<\alpha \leq 1)$. The truncation strategy is illustrated in Fig. 5 . These $\boldsymbol{W}_{h}$ coefficients located in the low-sequency triangular zone are retained while those $\boldsymbol{W}_{h}$ coefficients located in 


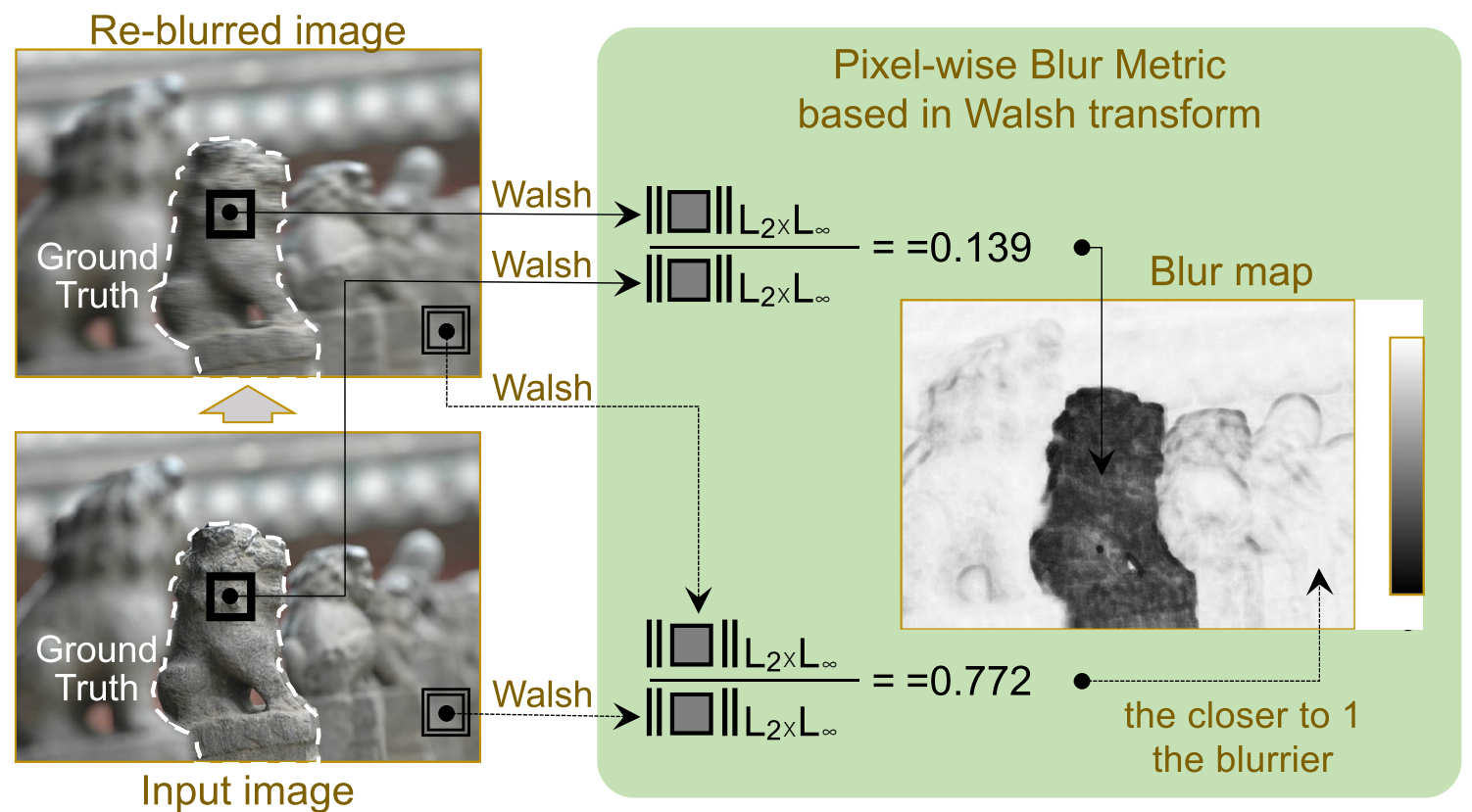

Fig. 3 The sketch of pixel-wise blur metric in sequency domain and the consequent blur map. The re-blur kernel we used here is the mean filter with a window size of $9 \times 9$

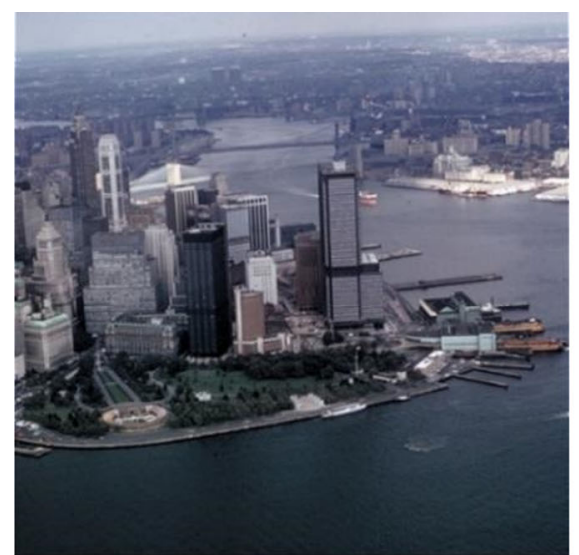

(a)

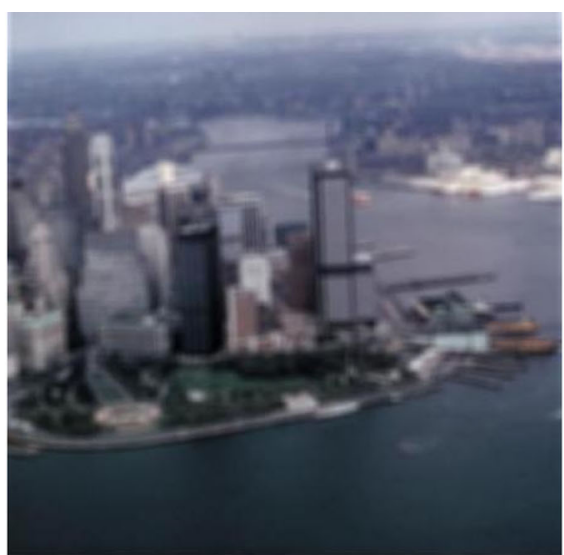

(b)

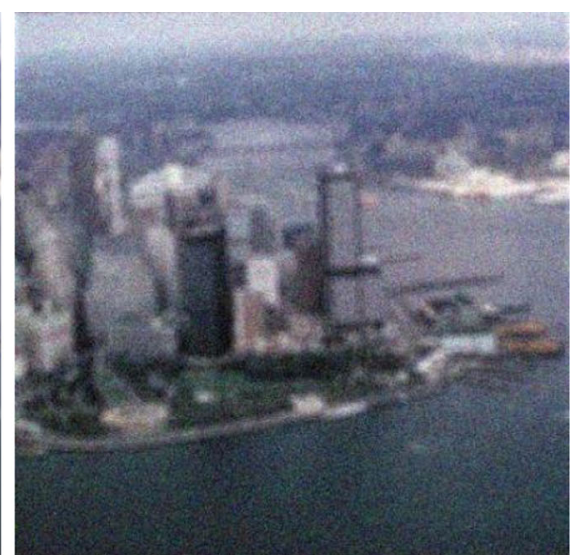

(c)
Fig. 4 Noise-induced blur estimation error. a Is a clear image with average blurriness $\lambda=0.172$ calculated by Eq. (5); $\mathbf{b}$ Is the re-blurred version of a using a $9 \times 9$ mean filter and its average blurriness $\lambda=0.749$; $\mathbf{c}$ is the noise-polluted version of $\mathbf{b}$ by adding the Gaussian noise of $\sigma=$

high-sequency zones are truncated and discarded (replaced by 0 ). It is obvious that the value of truncation proportion $(\alpha)$ should be adaptive to noise intensity (denoted by the standard deviation $\sigma$ ). To find the relation between $\alpha$ and $\sigma$, we have performed a series of experiments. First, five image samples from the CSIQ dataset [36] are chosen as original clear images. Then, we simulate their re-blurred versions with different blur kernels to obtain different levels of blurry images. Meanwhile, the noise-polluted versions of these blurry images are created by adding Gaussian white
$5 \mathrm{e}-2$, but its average blurriness presents a low value $\lambda=0.183$. Obviously, the noise-polluted blurry image is misjudged into a clear one by Eq. (5)

noises with different intensity. Then, for each noise-polluted blurry image, an appropriate $\alpha$ can be found via:

$\alpha_{\mathrm{opt}}=\underset{2 / N \leq \alpha \leq 1}{\arg \min }\left\{\left|\lambda\left(I^{b}\right)-\lambda^{l}\left(I^{b+n}\right)\right|\right\}$

where $\boldsymbol{I}^{b}$ denotes the noise-free blurry image and $\boldsymbol{I}^{b+n}$ denotes the noise-polluted blurry image.

Figure 6 shows some results of above-mentioned experiments. As it is observed, there is a fairly stable relation between the optimal truncation proportion $\alpha_{\text {opt }}$ and the noise 


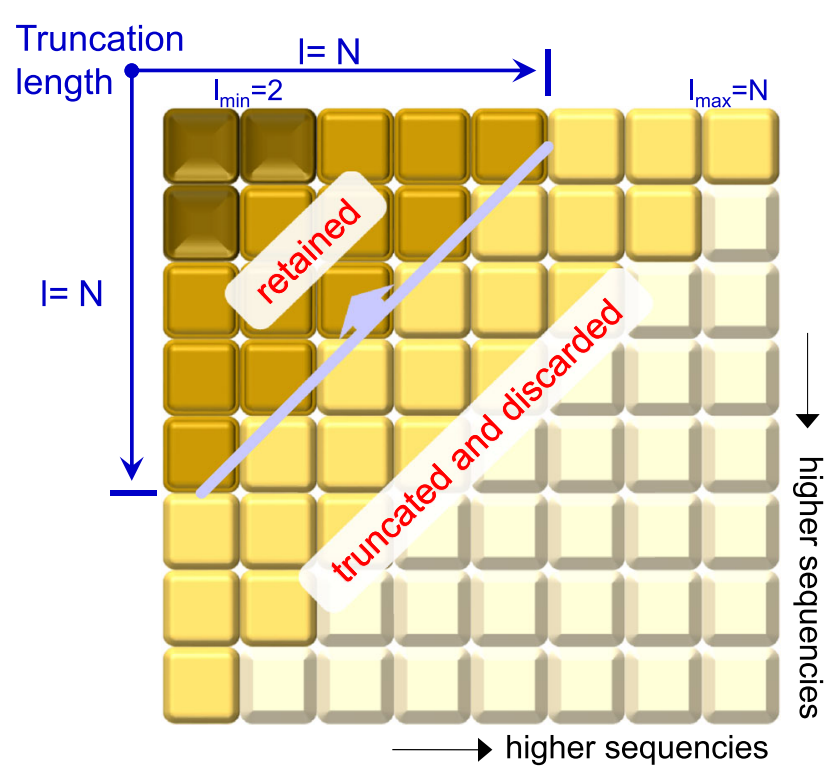

Fig. 5 The schematic of sequency spectrum truncation strategy. Lowsequency coefficients within a right-angled isosceles triangular zone are retained while other middle and high-sequency coefficients are truncated and discarded (replaced by 0 ) in the sequency spectrum. $l=\alpha N$ is the truncation length and $\alpha$ is an adaptive truncation proportion

intensity $(\sigma)$ under a certain noise-to-signal ratio (NSR). Therefore, it can be conceived that $\alpha_{\text {opt }}$ is dependent on both $\sigma$ and NSR. In order to determine the appropriate value of $\alpha_{\text {opt }}$ for an input image or patch, we must provide the relation among $\alpha, \sigma$, and NSR. This relation $(\boldsymbol{N})$ can be trained using a radial basis function (RBF) network and multiple sets of $\left\{\alpha_{\text {opt }}, \sigma, N S R\right\}$ acquired from experiments. Then, the trained RBF network $\left(N_{\mathrm{RBF}}\right)$ can be used to estimate the $\alpha_{\text {opt }}$ of the noise-polluted blurry image.

For a given image with unknown noise, we first employ the SOVNE algorithm [37] to estimate its noise intensity, i.e., the standard deviation $\sigma$. Subsequently, the noise-to-signal ratio can be obtained by the standard deviation ratio between noise and image: NSR $=\sigma / \sigma_{\text {image }}$. Then, the optimal truncation proportion $\alpha_{\text {opt }}$ is inferred via $N_{\mathrm{RBF}}(\sigma, \mathrm{NSR})$.

Then, we recalculate the average blurriness of Fig. $4 \mathrm{c}$ (noise-polluted) using Eq. (6) and obtain its blurriness value of $\lambda=0.744$. This value is quite close to that of the noise-free blurry image $(\lambda=0.749)$. It demonstrates that the truncation strategy can effectively eliminate the noise interference. On the other hand, we also use Eq. (6) to recalculate the average blurriness of Fig. $4 a, b$ (noise-free). The new values of $\lambda$ are 0.168 and 0.751 , respectively, which are very approximate to the results ( 0.172 and 0.749$)$ calculated by Eq. (5). It indicates that Eq. (6) with adaptive truncation strategy is appropriate for both noise-polluted and noise-free cases. Therefore, in this paper, we use Eq. (6) to estimate the blurriness of each pixel on its local patch and obtain the blur map of entire input image.

\section{Blur segmentation}

Besides pixel-wise blur estimation, another critical step for partial blur detection is blur segmentation, which divides the entire blur map $(\boldsymbol{\Lambda})$ into blur and non-blur regions for further processing like deblurring. Here, we propose a multi-scale fusion segmentation algorithm to accurately extract the blur region based on the clustering-guided region growth. The flowchart is shown in Fig. 7.

\section{Fast clustering}

The $k$-means clustering [34] is a classical unsupervised classification algorithm, which gathers similar elements based on the distance criteria. Considering the segmentation result is binary blur/non-blur, the number of clusters $(k)$ is exactly set to 2 . The maximum blurriness $\left(\lambda_{\max }\right)$ and minimum blurriness $\left(\lambda_{\min }\right)$ in the blur map $(\boldsymbol{\Lambda})$ are set as two initial cluster centers, respectively. Hence, we can implement a
Fig. 6 The relation between the optimal truncation proportion $\left(\alpha_{\text {opt }}\right)$ and noise intensity $(\sigma)$ for five image examples (from CSIQ dataset) at three noise levels (different NSR)

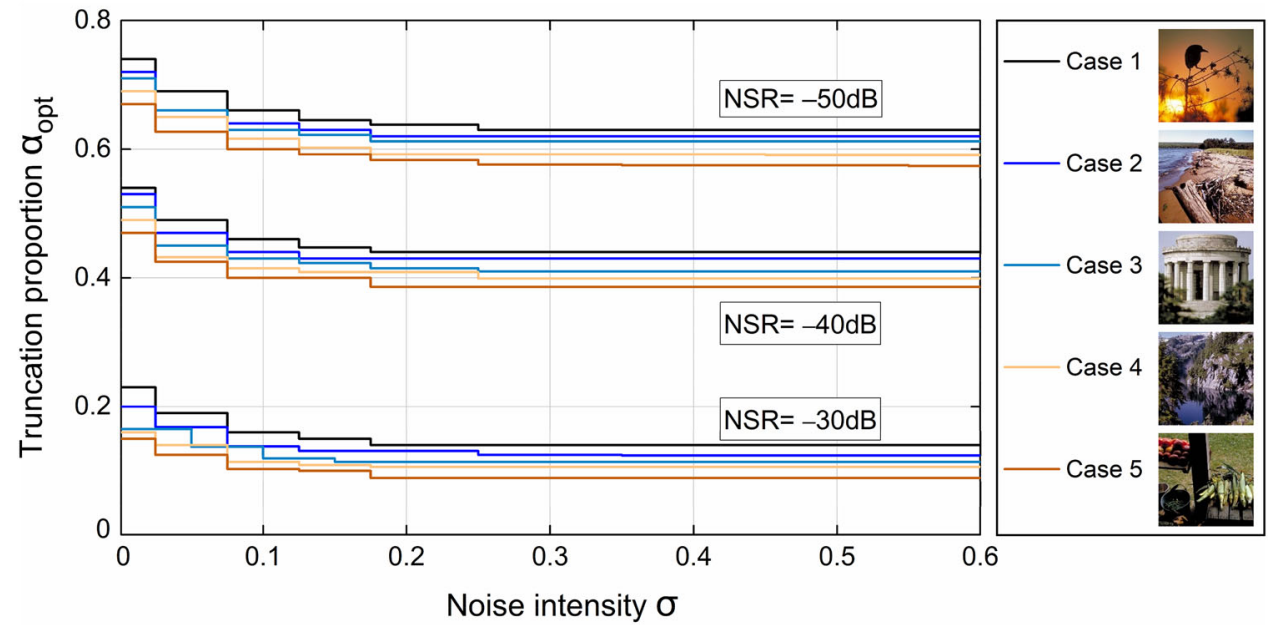




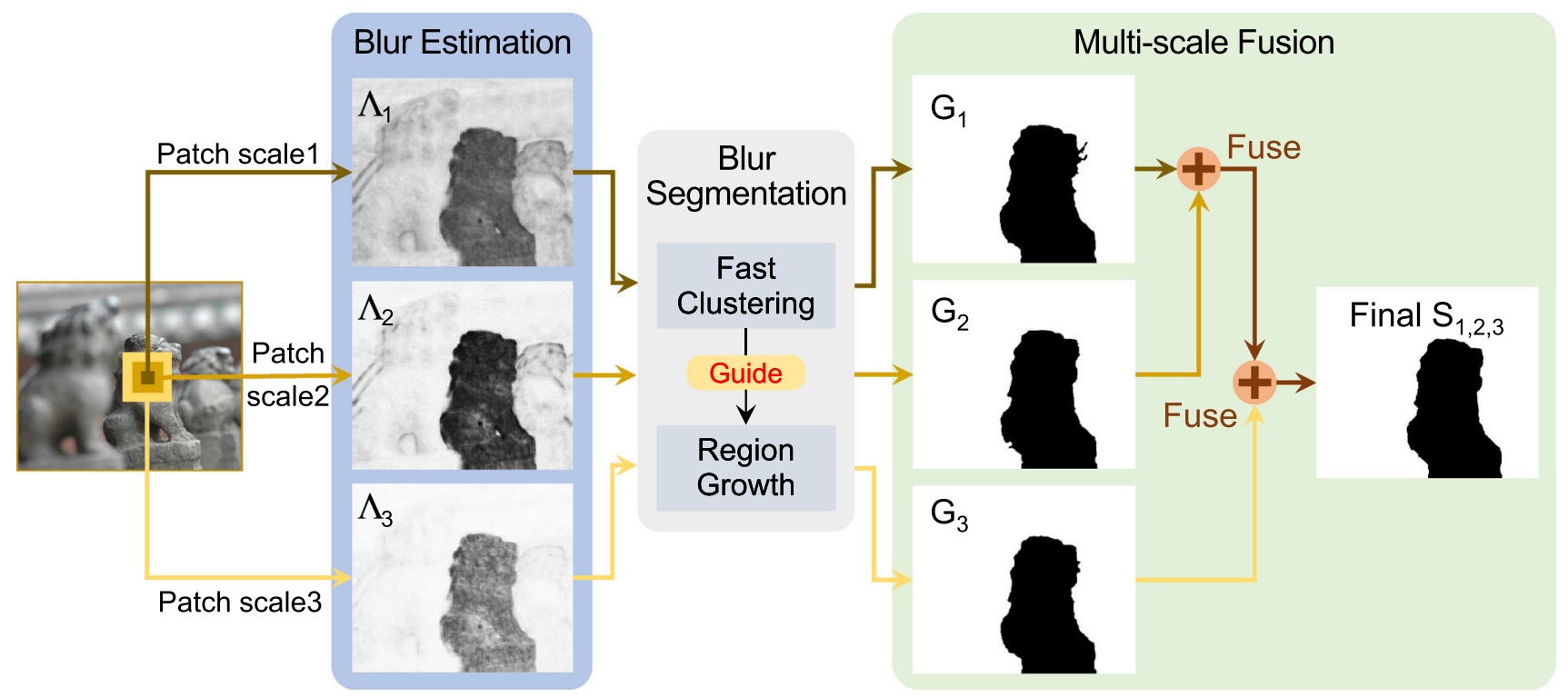

Fig. 7 The proposed segmentation framework integrated by clustering-guided region growth and multi-scale fusion

very fast $k$-means clustering $(k=2)$, as represented in Algorithm 1, to get a clustered map $(\boldsymbol{C})$.
Hence, we can obtain three blur maps $\left\{\boldsymbol{\Lambda}_{1}, \boldsymbol{\Lambda}_{2}, \boldsymbol{\Lambda}_{3}\right\}$ and three corresponding clustered maps $\left\{\boldsymbol{C}_{1}, \boldsymbol{C}_{2}, \boldsymbol{C}_{3}\right\}$. Then, as

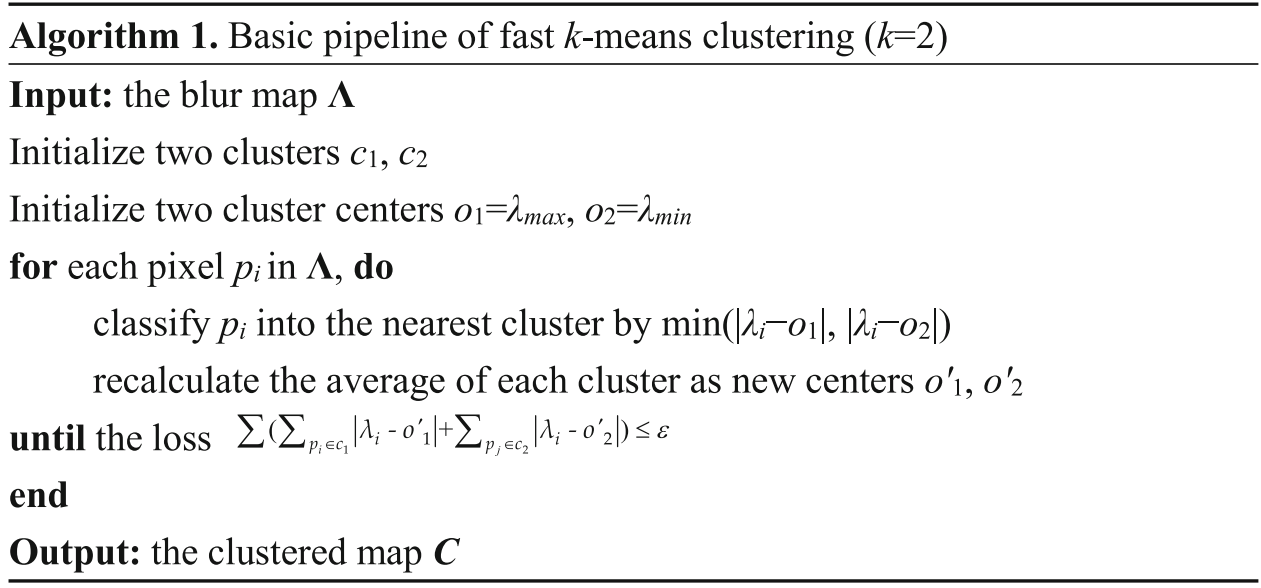

\section{Multi-scale region growth}

For the input image, we use Eq. (6) to estimate the blurriness of each pixel based on three local patches whose scales are $N_{1} \times N_{1}, N_{2} \times N_{2}, N_{3} \times N_{3}\left(N_{1}<N_{2}<N_{3}\right)$, respectively. 
Algorithm 2, we implement the region growth algorithm to get three grown maps $\left\{\boldsymbol{G}_{1}, \boldsymbol{G}_{2}, \boldsymbol{G}_{3}\right\}$ guided by three clustered maps $\left\{\boldsymbol{C}_{1}, \boldsymbol{C}_{2}, \boldsymbol{C}_{3}\right\}$.
Accordingly, we fuse $\boldsymbol{G}_{3}$ and $\boldsymbol{S}_{1,2}$ as above to merge scale3, and get the final segmentation map $\boldsymbol{S}_{1,2.3}$.

\begin{tabular}{l} 
Algorithm 2. Basic pipeline of region growing. \\
\hline Input: the clustered map $C$ \\
Initialize a seed $s$ in each main clustered region \\
for each seed $s$, do \\
$\quad$ for each neighbor $n_{s}$ of the eight-neighborhood, do \\
$\quad$ grow: transfer $n_{s}$ into a new seed if value $\left(n_{s}\right)=\operatorname{value}(s)$ \\
$\quad$ until none of neighbors can grow \\
end \\
until all seeds stop growing \\
end \\
Output: the grown map $\boldsymbol{G}$
\end{tabular}

\section{Multi-scale fusion}

It is well known that the blur degree is sensitive to scales [26, 30], which is definitely a tough problem in blur detection. To mitigate the scale sensitivity and heighten the segmentation accuracy, a multi-scale fusion strategy is used here to get the final segmentation map $\boldsymbol{S}$. The scale1 and scale2 are fused first. We compare the difference between $\boldsymbol{G}_{1}$ and $\boldsymbol{G}_{2}$ by $\boldsymbol{d}=$ $\boldsymbol{G}_{1} \oplus \boldsymbol{G}_{2}$, where $\oplus$ denotes the exclusion OR operation.

For a pixel $p_{x, y}$, the value of $\boldsymbol{d}\left(p_{x, y}\right)$ is 1 when and only when $\boldsymbol{G}_{1}\left(p_{x, y}\right) \neq \boldsymbol{G}_{2}\left(p_{x, y}\right)$, which indicates the class (blur or non-blur) of $p_{x, y}$ is different on scale1 and scale2. Hence, we redetermine the class of $p_{x, y}$ according to the major class in the eight neighborhood of this pixel:

$\mathrm{S}_{1 \ominus 2}\left(p_{x, y}\right)=\underset{f=0,1}{\arg \max }\left[\sum_{q \in \mathrm{n}_{8 \leftarrow \mathrm{p}}} \operatorname{Ind}\left(\mathrm{G}_{2}(q)=f\right)\right]$,

where $\boldsymbol{n}_{\mathbf{8} \leftarrow p}$ denotes the eight neighborhood of $p_{x, y}$ and $q$ denotes the neighbor therein. $f=0$ and 1 denote the nonblur class and blur class, respectively. $\operatorname{Ind}(\cdot)$ is an indicator function. Ind takes 1 when $\boldsymbol{G}_{2}(q)=f$ is true, otherwise it takes 0 .

If $\boldsymbol{G}_{1}\left(p_{x, y}\right)=\boldsymbol{G}_{2}\left(p_{x, y}\right)$, the value of $\boldsymbol{d}\left(p_{x, y}\right)$ will be 0 . At this point, $\boldsymbol{S}_{1,2}\left(p_{x, y}\right)=\boldsymbol{G}_{2}\left(p_{x, y}\right)$.

Therefore, the fusion result of $\boldsymbol{G}_{1}$ and $\boldsymbol{G}_{2}$ is:

$S_{1,2}\left(p_{x, y}\right)=\left\{\begin{array}{ll}\underset{f=0,1}{\arg \max }\left[\sum_{q \in n_{8 \leftarrow p}} \operatorname{Ind}\left(G_{2}(q)==f\right)\right], & d\left(p_{x, y}\right)=1 \\ G_{2}\left(p_{x, y}\right), & d\left(p_{x, y}\right)=0\end{array}\right.$.

\section{Experimental results}

In this section, we show experimental results of the proposed sequency-based blur detection method, as well as comparisons with previous state-of-the-art methods. To evaluate the performance, two publicly available datasets have been considered: CUHK [12] and DUT [26].

CUHK consists of 1000 partially blurred images captured from real world, wherein 704 images are out-of-focus and 296 images are motion blurred. DUT contains 1100 challenging images, all of which are partially out-of-focus natural images. All samples in both datasets are noise-free and annotated with corresponding ground-truth maps.

On this basis, we add a certain amount of Gaussian noise, speckle noise, and impulsive noise into CUHK and DUT to simulate real noise pollution. The noise intensity (standard deviation) takes from 0.01 to 0.5 at an interval of 0.05 , which generates a noise-polluted version of CUHK and DUT.

To objectively validate the proposed method, we choose four widely used indicators: $p$ (precision), $r$ (recall), $F_{\beta}$ ( $F_{\beta}$-measure), and MAE (mean absolute error). Precision is a measure of the ability of the method to recognize only blur regions. Recall indicates the ability of the method to identify all blur regions. $\mathrm{F}_{\beta}$-measure is a comprehensive weighted indicator of precision and recall. Here we use $\beta=1$ that means precision and recall has the equal importance. MAE is an error-related indicator computed by comparing the final segmentation map (S) and the ground-truth map $(\boldsymbol{G T})$. For $p, r$, and $F_{1}$, the 
Table 1 Definitions of evaluation indicators

\begin{tabular}{ll}
\hline Indicator & Formula \\
\hline$P$ & $\boldsymbol{R} \cap \boldsymbol{R}_{G T} / \boldsymbol{R}$ \\
$R$ & $\boldsymbol{R} \cap \boldsymbol{R}_{G T} / \boldsymbol{R}_{G T}$ \\
$F_{\beta}$ & $\left(1+\beta^{2}\right) \bullet \bullet \bullet r /\left(\beta^{2} \bullet p+r\right)$ \\
MAE & $(1 / W \bullet H) \Sigma \Sigma|\boldsymbol{S}-\boldsymbol{G T}|$ \\
\hline
\end{tabular}

$\boldsymbol{R}$ is the detected blur region; $\boldsymbol{R}_{G T}$ is the labeled blur region in $\boldsymbol{G T}$ $W, H$ is width and height of the input image, respectively

higher value indicates the better performance, but $M A E$ acts the opposite. All indicators are defined in Table 1.

\section{Implementation}

In our experiments, we use a $9 \times 9$ mean filter as the re-blur kernel for the sake of efficiency, and select $N=4,8$, and 16 as three patch scales in pixel-wise blur estimation. Here, we experimented various patch scales and achieved the best performance when patch sizes are 4,8 , and 16 . For arbitrary input image with unknown noise, the SOVNE algorithm [37] is employed to estimate the noise intensity. A pre-trained RBF network $\left(N_{\mathrm{RBF}}\right)$ is used to determine the optimal truncation proportion $\alpha_{\text {opt }}$. Then, we can estimate the blur map by Eq. (6) and produce the final segmentation map by Eq. (13). Our method is implemented in MATLAB with C-Mex compilation, and our experiments are performed on a workstation with Core i7 3.50 GHz CPU and 16 GB RAM. Some exemplar blur maps and segmentation maps are shown in Fig. 8.

\section{Comparisons}

The first series of experiments aim to compare the proposed method with the state-of-the-art methods in recent five years, including JNDB [13], SSA [15], LBP [17], DCT [20], HiFST [6], DHDE [24], BDNet [25], CFLLM [27], BTBCRL [30], and DeFusionNET [38], where the latter five methods are based on deep learning. Considering that some methods [13, $17,24,27,30,38]$ are designed only for defocus blur, the comparisons are limited to defocus blur images (and their noise-polluted versions). In this section, we use the implementations provided by authors or the results reported in literatures without any modification.

\section{Visual comparison}

Figure 9 shows a visual comparison of all involved blur detection methods on common cases and challenging cases, where the challenging case 1 contains two images (CUHK) with cluttered backgrounds, the challenging case 2 contains two images (DUT) with homogeneous regions, and the challenging case 3 contains two images (DUT) with similar foreground and background. It can be observed that our method and DeFusionNET are the two methods that yield the most accurate segmentation map in common and challenging cases, benchmarked by the ground truth. The third-place BTBCRL performs well in challenging cases with cluttered backgrounds, but perform less well in challenging cases with homogeneous regions or similar fore/background. It is also worth mentioning that the segmented blur/sharp region by our method has a high internal connectivity.

\section{Quantitative comparison}

Table 2 presents the compared results of $F_{1}$ and MAE from different methods on different types of samples. Hereinto, noise-free samples are from $\mathrm{CUHK}_{1}$ (the out-of-focus part of CUHK), $\mathrm{CUHK}_{2}$ (the motion-blurred part of CUHK), and DUT; noise-polluted samples are from NCD. It can be seen that our method achieves the top performance on $\mathrm{CUHK}_{2}$ and NCD, and ranks second on $\mathrm{CUHK}_{1}$ and DUT.

On noise-free $\mathrm{CUHK}_{1}$, our method achieves a $F_{1}$-measure of 0.899 and a MAE value of 0.094 , which ranks second only to deep learning-based BTBCRL $\left(F_{1}=0.904\right.$ and MAE $=0.088$ ). On noise-free $\mathrm{CUHK}_{2}$, our method ranks first in $F_{1}$-measure and MAE whose values are 0.884 and 0.102 , respectively. Compared with the second-best DCT, our method improves the $F_{1}$-measure by $2.8 \%$ and reduces the MAE by $30.6 \%$. On noise-free DUT, our method achieves the second-highest $F_{1}$-measure of 0.881 and the second-lowest MAE value of 0.105 , behind deep learning-based DeFusion$\operatorname{NET}\left(F_{1}=0.889\right.$ and MAE $\left.=0.099\right)$.

For noised samples from the NCD dataset, the proposed method performs distinctly better than other competitive methods. Our method achieves the highest $F_{1}$-measure of 0.873 and the lowest MAE value of 0.118 . Compared with the second-place BTBCRL, the $F_{1}$-measure increases by $15.3 \%$ and the MAE decreases by $44.3 \%$.

Moreover, we test the pre-denoising technique in our experiments. The BM3D algorithm [41, 42], one of SOTA denoising algorithms, are used to denoise these noisepolluted samples. The results of $F_{1}$ and MAE on denoised samples are presented in the bottom of Table 2. Obviously, we can observe a performance increase by applying a predenoising processor to different methods. For our proposed method, the result also improves after denoising. It demonstrates the pre-denoising technique is an effective means to mitigate the noise sensitivity, although it introduces extra computing costs. However, even with the pre-denoising process, the performance on noise-polluted images is still lower than that on noise-free images. It is probably because that the pre-denoising technique could remove useful sharp information while filtering harmful noises. 


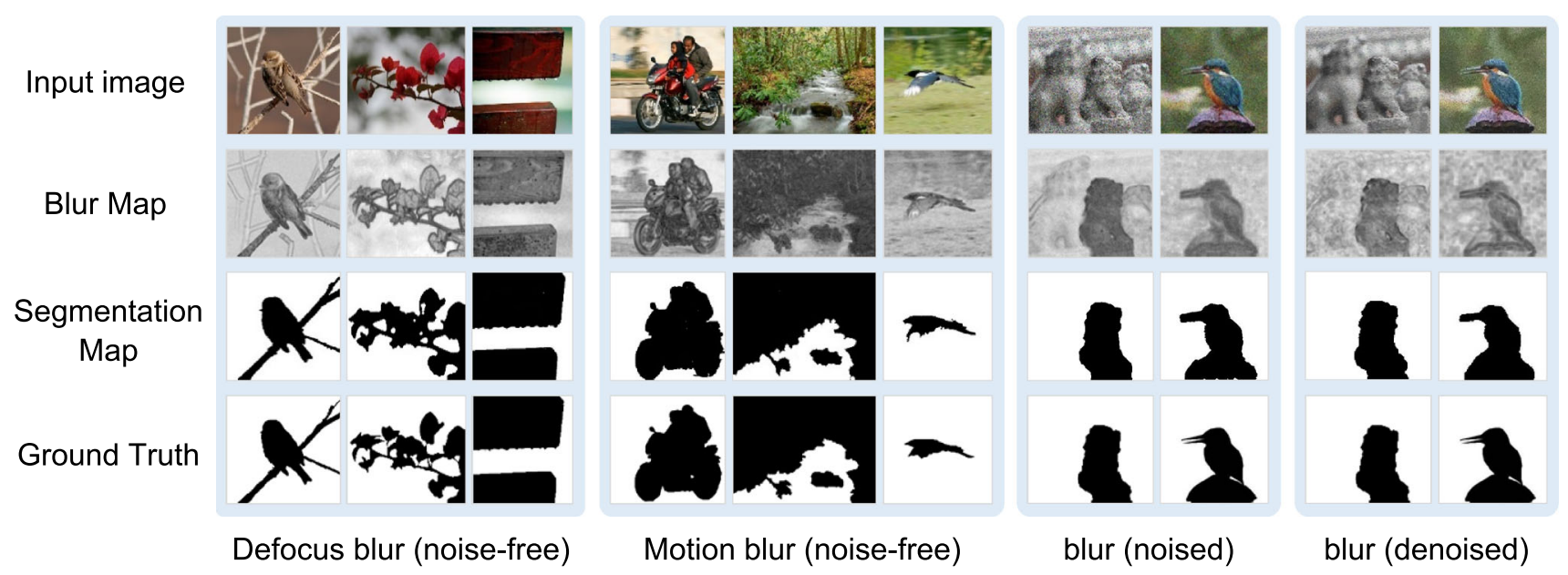

Fig. 8 Some exemplar blur maps and segmentation maps by the proposed method in noise-free and noise-polluted conditions including defocus and motion

Table 2 Quantitative comparison of $F_{1}$ and MAE among different methods. The first and second places in each sample type are highlighted in bold and italics, respectively

\begin{tabular}{|c|c|c|c|c|c|c|c|c|c|c|c|c|}
\hline Sample type & Ind & JNDB & SSA & LBP & DCT & HiFST & DHDE & BDNet & CFLLM & DeFusion NET & BTBCRL & Ours \\
\hline \multirow[t]{2}{*}{ Defocus $\left(\mathrm{CUHK}_{1}\right)$} & $F_{1}$ & 0.699 & 0.770 & 0.807 & 0.875 & 0.717 & 0.712 & 0.841 & 0.836 & 0.895 & 0.904 & 0.899 \\
\hline & MAE & 0.352 & 0.203 & 0.183 & 0.122 & 0.233 & 0.237 & 0.209 & 0.161 & 0.099 & 0.088 & 0.094 \\
\hline \multirow[t]{2}{*}{ Motion $\left(\mathrm{CUHK}_{2}\right)$} & $F_{1}$ & - & 0.761 & - & 0.860 & 0.712 & - & 0.832 & - & - & - & 0.884 \\
\hline & MAE & - & 0.210 & - & 0.137 & 0.239 & - & 0.162 & - & - & - & 0.102 \\
\hline \multirow[t]{2}{*}{ Defocus (DUT) } & $F_{1}$ & 0.628 & 0.751 & 0.780 & 0.768 & 0.688 & 0.627 & - & - & 0.889 & 0.854 & 0.881 \\
\hline & MAE & 0.370 & 0.193 & 0.211 & 0.228 & 0.318 & 0.401 & - & - & 0.096 & 0.142 & 0.105 \\
\hline \multirow[t]{2}{*}{ Avg } & $F_{1}$ & 0.664 & 0.761 & 0.794 & 0.834 & 0.706 & 0.670 & 0.837 & 0.836 & 0.893 & 0.879 & 0.887 \\
\hline & MAE & 0.361 & 0.202 & 0.197 & 0.162 & 0.263 & 0.319 & 0.186 & 0.161 & 0.097 & 0.115 & 0.101 \\
\hline \multirow[t]{2}{*}{ Noised (NCD) } & $F_{1}$ & 0.539 & 0.643 & 0.664 & 0.655 & 0.623 & 0.611 & - & - & - & 0.757 & 0.873 \\
\hline & MAE & 0.438 & 0.380 & 0.329 & 0.354 & 0.379 & 0.414 & - & - & - & 0.212 & 0.118 \\
\hline \multirow[t]{2}{*}{ Denoised (NCD) } & $F_{1}$ & 0.635 & 0.717 & 0.738 & 0.772 & 0.678 & 0.654 & - & - & - & 0.874 & 0.879 \\
\hline & MAE & 0.375 & 0.244 & 0.212 & 0.202 & 0.336 & 0.372 & - & - & - & 0.118 & 0.113 \\
\hline
\end{tabular}

Avg. and Ind. are the abbreviation of indicator and average, respectively

Avg. is a rough value only for reference because some of these methods do not share the same statistical range

\section{Runtime comparison}

We also report the average runtime of competitive blur detection methods for one single input image in Table 3. For some methods [25, 27, 30, 38, 39] whose implementations are unavailable, we use the runtime reported in their papers. For the rest, we use their implementations on our workstation (i7 CPU and 16 GB RAM). As it is observed, our method takes about 0.7 s only using CPU to process an input image, behind DeFusionNET [38] and $\mathrm{R}^{2} \mathrm{MRF}$ [39] but faster than other competitive methods. Besides, our method needs about $0.5 \mathrm{~s}$ to pre-train the RBF network, which is far faster than deep learning-based BTBCRL [30] (about 5 days), DeFusionNET (about $11.7 \mathrm{~h}$ ), and $\mathrm{R}^{2} \mathrm{MRF}$ (about $0.8 \mathrm{~h}$ ). Note that our method has a lightweight architecture that requires neither parallel computing resources nor massive model parameters. Hence, it has the potential to be applied in portable devices or embedded platforms.

In summary, the proposed sequency-based blur detection method is effective and efficient in different blur scenarios (including out-of-focus and motion) and various challenging cases (containing cluttered backgrounds, homogeneous regions, and similar fore/background). By the above comparisons, our method presents a good detection performance and strong noise robustness, which indicates the superiority to other competitive methods. 


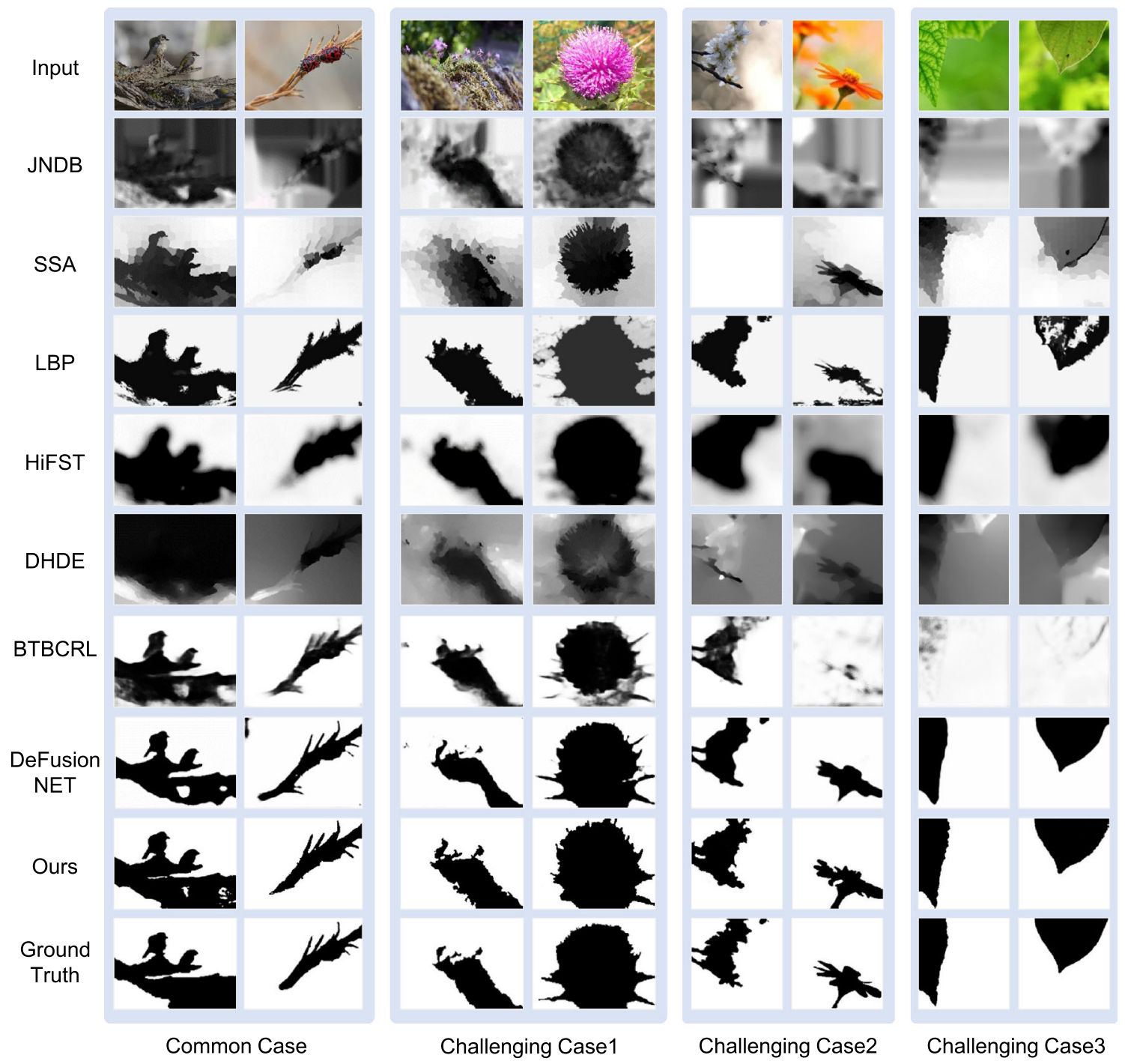

Fig. 9 Visual comparison of blur segmentation results from different methods including JNDB [13], SSA [15], LBP [17], HiFST [6], DHDE [24], BTBCRL [30], DeFusionNET [38], and ours. Challenging case1 has cluttered backgrounds, case 2 has homogeneous regions, and case 3 has similar foreground and background

\section{Discussion}

\section{Necessity of Walsh transform}

In this paper, the Walsh transform is introduced into our blur metric. This is mainly based on two following concerns. (1) The Walsh basis is sparse with only \pm 1 values. In other words, the Walsh transform involves only addition/subtraction and no multiplication/division, which can be easily compiled by computer. Coupled with the simple recursive relationship of Hadamard sequence, the Walsh transform has a great efficiency advantage that its computing cost and required storage are far lower than other transforms like Fourier. (2) The Walsh transform is a complete orthogonal transform with the property of energy compaction which always compresses image information to the low-sequency spectrum zone. Therefore, a few low-sequency $\boldsymbol{W}_{h}$ coefficients can effectively represent rich image information and meanwhile protect against high-sequency noise interference [23]. Due to the advantages of computation rapidity and energy compaction, the Walsh transform has been widely used in many fields such as compressed sensing [31], coding and decoding $[32,33]$. Our experimental results demonstrate it can be also applied in noise-immune blur detection. 
Table 3 Quantitative comparison of average runtime among different methods on one single image. The pre-training time is also shown in round brackets for some supervised methods and ours

\begin{tabular}{lllllllllllll}
\hline Method & JNDB & SSA & LBP & DCT & HiFST & DHDE & BDNet & CFLLM & BTBCRL & $\begin{array}{c}\text { Defusion } \\
\text { NET }\end{array}$ & $R^{2}$ MRF & Ours \\
\hline $\begin{array}{c}\text { GPU } \\
\text { require }\end{array}$ & No & No & No & No & No & Yes & Yes & Yes & Yes 1080Ti & Yes & Yes 1080Ti & No \\
$\begin{array}{l}\text { Image size } \\
\text { Runtime }\end{array}$ & $512^{2}$ & $512^{2}$ & $512^{2}$ & $512^{2}$ & $512^{2}$ & $512^{2}$ & $640 \times 480$ & $640 \times 457$ & $320^{2}$ & $320^{2}$ & $320^{2}$ & $512^{2}$ \\
& $13.8 \mathrm{~s}$ & $2.8 \mathrm{~s}$ & $26.5 \mathrm{~s}$ & $84.4 \mathrm{~s}$ & $95.2 \mathrm{~s}$ & $26.1 \mathrm{~s}$ & $45.2 \mathrm{~s}$ & $5.3 \mathrm{~s}$ & $12.0 \mathrm{~s}(5 \mathrm{~d})$ & $0.06 \mathrm{~s}$ & $0.09 \mathrm{~s}$ & $0.7 \mathrm{~s}(0.5 \mathrm{~s})$ \\
\end{tabular}

Table 4 Results of the ablation experiment on truncation strategy

\begin{tabular}{llllll}
\hline Our algorithm & \multicolumn{2}{l}{ Noised DUT } & & \multicolumn{2}{l}{ Noised CUHK } \\
\cline { 2 - 3 } \cline { 6 - 7 } & $F_{1}$ & MAE & & $F_{1}$ & MAE \\
\hline With truncation & 0.870 & 0.122 & & 0.875 & 0.121 \\
Without truncation & 0.568 & 0.392 & & 0.595 & 0.381 \\
\hline
\end{tabular}

\section{Ablation study}

This section aims to evaluate the effect of two strategies: (i) the sequency spectrum truncation strategy for noise-immune blur estimation; (ii) the multi-scale fusion strategy for fine blur segmentation. To this end, two ablation experiments are conducted on DUT, CUHK, and NCD datasets.

\section{Sequency spectrum truncation strategy}

To validate the anti-noise effectiveness of this strategy, we set an ablation experiment on NCD using two algorithms: the complete version of our algorithm and the ablated version by removing only the truncation step. Their performances are reported in Table 4. We can observe that the algorithm equipped with truncation strategy achieves a far better performance than the algorithm with no truncation. It demonstrates that the sequency spectrum truncation strategy plays a vital role on the noise immunity of our method.

\section{Multi-scale fusion strategy}

The proposed method fuses three patch scales of $4 \times 4,8 \times 8$, and $16 \times 16$. To prove the contribution of multi-scale fusion strategy, we set an ablation experiment including three algorithms: the complete algorithm using all three patch scales, the ablated algorithm using two patch scales of $8 \times 8$ and $16 \times 16$, and the ablated algorithm using one patch scale of $16 \times 16$. The corresponding $F_{1}$ and MAE of three algorithms on DUT, CUHK, and NCD are reported in the upper part of Table 5. It can be seen that the complete algorithm evidently outperforms other two ablated algorithms, which demonstrates the effectiveness of the proposed multi-scale fusion strategy. Moreover, we conduct experiments using different fusion orders of three scales. The results are reported in the lower part of Table 5 , where scale 1 , scale 2 , and scale 3 denote $4 \times 4,8 \times 8$, and $16 \times 16$, respectively. We can see that there is little difference in detection performance among different fusion orders. Mathematically, the order of (scale1 + scale2) + scale 3 looks slightly better on $F_{1}$ and MAE than two other orders. Therefore, in this paper, we merge scale 1 and scale 2 at first, then fuse scale3.

\section{Noise immunity}

Table 6 reports the results of $F_{1}$-measure and $M A E$ of our method at different noise levels. It shows that our method has a good anti-noise capacity and maintains a relatively high detection performance. However, with the increase of noise level, the anti-noise capacity will also be moderately reduced. In the bottom of Table 6, we also give the updated results of $F_{1}$-measure and $M A E$ after adding a BM3D pre-denoising processor. It can be observed that the proposed method combined with BM3D provides a higher detection performance, especially facing large noise. It demonstrates that the applying of the pre-denoising technique can enhance the anti-noise capacity of our method, although it introduces extra computing costs.

\section{Failure cases}

There are some knotty situations that can cause the proposed method to fail. The first one is the ambiguity between blur region and textureless sharp region. As shown in Fig. 10, our method has difficulty telling the difference between the smooth blur region (marked by blue rectangle) and the smooth sharp region (marked by red rectangle), because they have similar smoothness with few textures. Actually, this problem is fairly challenging for any blur detection algorithm. Another knotty situation is the interference of strong image noise. As shown in Fig. 11, when the noise variance/density achieves the level of $5 \mathrm{e}-1$, the blur map becomes indistinguishable; hence, the proposed method fails to segment the correct blur/sharp region. This noise- 


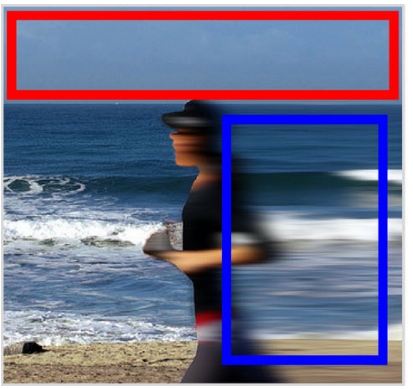

Input image

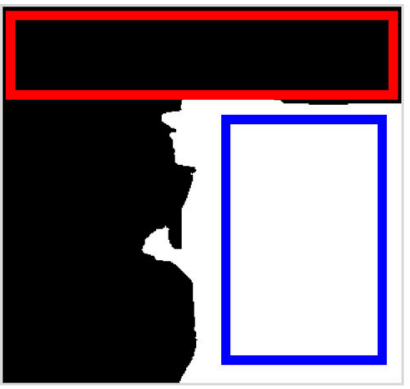

Ground Truth

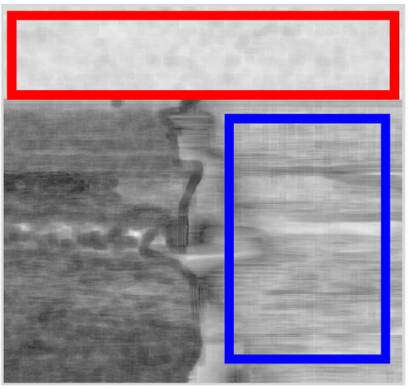

Blur map

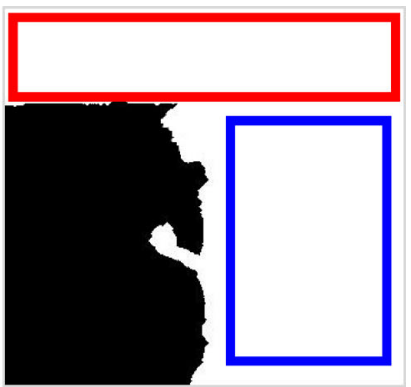

Segmentation map

Fig. 10 Failure case due to the ambiguity between blur region (marked by blue rectangle) and textureless sharp region (marked by red rectangle)

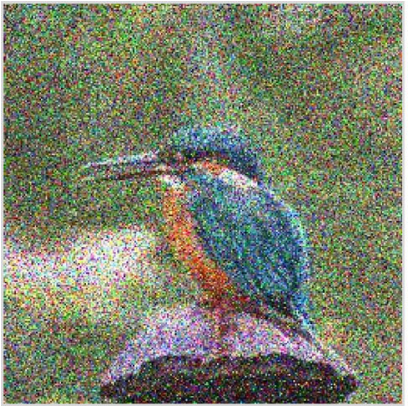

Input image

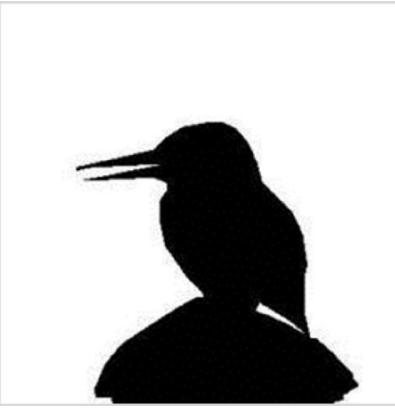

Ground Truth



Blur map

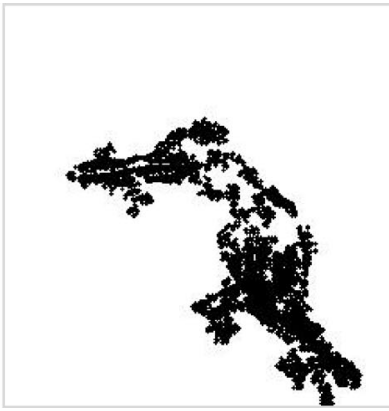

Segmentation map

Fig. 11 Failure case due to strong noise with a variance/density of 5e-1

induced failure can be mitigated by applying an effective pre-denoising technique as mentioned in "Quantitative comparison".

\section{Conclusions}

In this work, we propose a novel noise-immune blur detection method. First, a simple re-blur scheme is used to inspect the unknown blur in an input image. Then, we design a pixel-wise blurriness metric based on the sequency spectrum derived by the Walsh transform. Meanwhile, to solve the misdetection problem caused by image noises, we develop an adaptive sequency spectrum truncation strategy to eliminate the noise interference. By combining the re-blur scheme, sequency transform, and adaptive truncation, we can achieve an effective and noise-robust blur detection that produces a fine blur map. Finally, the blur region is accurately segmented using the clustering-guided region growth framework and the multi-scale fusion strategy. Experimental results on two benchmark datasets (DUT and CUHK) and their noisepolluted versions (Noised DUT and CUHK) demonstrate the superiority of our method to competitive blur detection approaches. The proposed method achieves the top perfor-
Table 5 Results of the ablation experiment on multi-scale fusion strategy

\begin{tabular}{|c|c|c|c|c|c|c|}
\hline \multirow[t]{2}{*}{ Multi-scale fusion strategy } & \multicolumn{2}{|c|}{ CUHK } & \multicolumn{2}{|l|}{ DUT } & \multicolumn{2}{|l|}{ NCD } \\
\hline & $F_{1}$ & MAE & $F_{1}$ & MAE & $F_{1}$ & MAE \\
\hline \multicolumn{7}{|l|}{ Scale } \\
\hline Scale $=\{16\}$ & 0.883 & 0.118 & 0.866 & 0.131 & 0.849 & 0.164 \\
\hline Scales $=\{8,16\}$ & 0.886 & 0.107 & 0.871 & 0.120 & 0.861 & 0.136 \\
\hline Scales $=\{4,8,16\}$ & 0.892 & 0.099 & 0.881 & 0.105 & 0.873 & 0.118 \\
\hline \multicolumn{7}{|l|}{ Fusion order } \\
\hline$($ scale $1+$ scale 2$)+$ scale 3 & 0.892 & 0.099 & 0.881 & 0.105 & 0.873 & 0.118 \\
\hline$($ scale $1+$ scale 3$)+$ scale 2 & 0.892 & 0.101 & 0.880 & 0.106 & 0.871 & 0.120 \\
\hline$($ scale $2+$ scale 3$)+$ scale 1 & 0.891 & 0.102 & 0.881 & 0.106 & 0.872 & 0.118 \\
\hline
\end{tabular}

The order of $(\mathrm{A}+\mathrm{B})+\mathrm{C}$ means to merge $\mathrm{A}$ and $\mathrm{B}$ at first and then fuse $\mathrm{C}$ 
Table $6 F_{1}$-measure and $M A E$ of the proposed method at different noise levels

\begin{tabular}{|c|c|c|c|c|c|c|c|}
\hline \multicolumn{2}{|c|}{ Noise level } & $1 e-6$ & $1 \mathrm{e}-5$ & $1 \mathrm{e}-4$ & $1 e^{-3}$ & $1 \mathrm{e}-2$ & $1 \mathrm{e}-1$ \\
\hline \multicolumn{2}{|c|}{ One example } & & & $6-1$ & & & 48 \\
\hline \multirow{2}{*}{ Indicators } & $F_{1}$ & 0.877 & 0.872 & 0.867 & 0.854 & 0.813 & 0.764 \\
\hline & $M A E$ & 0.113 & 0.119 & 0.125 & 0.135 & 0.184 & 0.211 \\
\hline \multirow{2}{*}{$\begin{array}{l}\text { Indicators } \\
(+ \text { denoise })\end{array}$} & $F_{1}$ & 0.884 & 0.878 & 0.875 & 0.871 & 0.863 & 0.850 \\
\hline & $M A E$ & 0.105 & 0.113 & 0.116 & 0.121 & 0.130 & 0.144 \\
\hline
\end{tabular}

mance in DUT and the motion blur part of CUKH. It ranks second in the defocus blur part of CUHK. Especially for noise-polluted blurry images, the proposed method outperforms other competitors much and presents a prominent noise immunity, which has great application prospect in realistic noisy conditions. In terms of the runtime, our method is inferior only to a recent deep learning-based approach, but with higher accuracy and significantly shorter pre-training time. To summarize the above results, we believe that our method achieves the best balance between cost and benefit among all competitive methods.

As future work, we plan to explore a more adaptive truncation strategy, develop a more effective multi-scale fusion algorithm, and apply the proposed method into the blur detection of UAV-based and SAR-based images. In addition, we also plan to build a larger and richer dataset for expanding the blur detection filed.

Acknowledgements This research was funded by the National Natural Science Foundation of China $(62003227,52102467)$, the Independent Fund from the State Key Laboratory of Mechanical Behavior and System Safety of Traffic Engineering Structures (ZZ2021-12), the Natural Science Foundation of Hebei Province (F2021210016, E2021210011), and the Science Research Project of the Education Department of Hebei Province (QN2019066, QN2021135). We also thank the valuable comments and suggestions from the anonymous reviewers for improving the manuscript.

Funding This research was funded by the National Natural Science Foundation of China (62003227, 52102467), the Independent Fund from the State Key Laboratory of Mechanical Behavior and System Safety of Traffic Engineering Structures (ZZ2021-12), the Natural Science Foundation of Hebei Province (F2021210016, E2021210011), and the Science Research Project of the Education Department of Hebei Province (QN2019066, QN2021135).

Availability of data and material The datasets employed in this paper are publicly available.

Code availability The code is available from the corresponding author upon reasonable request.

\section{Declarations}

Conflict of interest The authors declare no conflict of interest.

Open Access This article is licensed under a Creative Commons Attribution 4.0 International License, which permits use, sharing, adaptation, distribution and reproduction in any medium or format, as long as you give appropriate credit to the original author(s) and the source, provide a link to the Creative Commons licence, and indicate if changes were made. The images or other third party material in this article are included in the article's Creative Commons licence, unless indicated otherwise in a credit line to the material. If material is not included in the article's Creative Commons licence and your intended use is not permitted by statutory regulation or exceeds the permitted use, you will need to obtain permission directly from the copyright holder. To view a copy of this licence, visit http://creativecomm ons.org/licenses/by/4.0/.

\section{References}

1. Yang D, Qin S (2015) Restoration of degraded image with partial blurred regions based on blur detection and classification. In: Proceedings of IEEE international conference on mechatronics and automation (ICMA). IEEE Computer Society Press, Los Alamitos, pp 2414-2419

2. Li L, Lin W, Wang X, Yang G, Bahrami K, Kot A (2017) Noreference image blur assessment based on discrete orthogonal moments. IEEE Trans Cybern 46(1):39-50

3. Li S, Fan X, Zhu B, Cheng Z (2017) A method for small infrared targets detection based on the technology of motion blur recovery. Acta Opt Sin 37(06):73-79

4. Lin J, Ji X, Xu W, Dai Q (2013) Absolute depth estimation from a single defocused image. IEEE Trans Image Process 22(11):4545-4550

5. Karaali A, Jung CR (2016) Image retargeting based on spatially varying defocus blur map. In: Proceedings of IEEE International Conference on Image Processing (ICIP). IEEE Computer Society Press, Los Alamitos, pp 2693-2697

6. Golestaneh SA, Karam LJ (2017) Spatially-varying blur detection based on multiscale fused and sorted transform coefficients of gradient magnitudes. In: Proceedings of IEEE conference on computer vision and pattern recognition (CVPR). IEEE Computer Society Press, Los Alamitos, pp 5800-5809

7. Zhou B, Han Y, Xu M, Li W, Pei Y, Lv P, Zhou L (2016) A fast nonlocal means image denoising algorithm. J Comput Aided Design Comput Graph 028(008):1260-1268 
8. Ali U, Mahmood M (2018) Analysis of blur measure operators for single image blur segmentation. Appl Sci 8(5):807

9. Liu R, Li Z, Jia J (2008) Image partial blur detection and classification. In: Proceedings of IEEE conference on computer vision and pattern recognition (cVPR). IEEE Computer Society Press, Los Alamitos, pp 954-961

10. Su B, Lu S, Tan CL (2011) Blurred image region detection and classification. In: Proceedings of IEEE conference on computer vision and pattern recognition (CVPR). IEEE Computer Society Press, Los Alamitos, pp 1397-1400

11. Lee H, Kim C (2014) Blurred image region detection and segmentation. In: Proceedings of IEEE international conference on image processing (ICIR). IEEE Computer Society Press, Los Alamitos, pp 4427-4431

12. Shi J, Xu L, Jia J (2014) Discriminative blur detection features. In: Proceedings of IEEE conference on computer vision and pattern recognition (CVPR). IEEE Computer Society Press, Los Alamitos, pp 2965-2972

13. Shi J, Xu L, Jia J (2015) Just noticeable defocus blur detection and estimation. In: Proceedings of IEEE conference on computer vision and pattern recognition (CVPR). IEEE Computer Society Press, Los Alamitos, pp 657-665

14. Harmuth HF, Ahmed N (1977) Sequency theory: foundations and applications. IEEE Trans Syst Man Cybern 9(5):312-312

15. Tang C, Wu J, Hou Y, Wang P, Li W (2016) A spectral and spatial approach of coarse-to-fine blurred image region detection. IEEE Signal Process Lett 23(11):1652-1656

16. Zhang X, Wang R, Jiang X, Wang W, Gao W (2016) Spatially variant defocus blur map estimation and deblurring from a single image. J Vis Commun Image Represent 35:257-264

17. Yi X, Eramian M (2016) LBP-based segmentation of defocus blur. IEEE Trans Image Process 25(4):1626-1638

18. Javaran TA, Hassanpour H, Abolghasemi V (2017) Automatic estimation and segmentation of partial blur in natural images. Vis Comput 33(2):151-161

19. Xu G, Quan Y, Ji H (2017) Estimating defocus blur via rank of local patches. In: Proceedings of IEEE international conference on computer vision (ICCV). IEEE Computer Society Press, Los Alamitos, pp 5371-5379

20. Wang X, Liang X, Zheng J, Zhou H (2017) No-reference detection and segmentation of partial blur for natural images. J Comput Aided Design Comput Graph 029(011):1980-1988

21. Xiao H, Lu W, Li R, Zhong N, Yeung Y, Chen J, Xue F, Sun W (2019) Defocus blur detection based on multiscale SVD fusion in gradient domain. J Vis Commun Image Represent 59:52-61

22. Liu S, Liao Q, Xue JH, Zhou F (2020) Defocus map estimation from a single image using improved likelihood feature and edge-based basis. Pattern Recognit 107:107485

23. Huang YS, Hsu CY (1991) Tracking moving objects in noisy environments using Walsh transform. Electron Lett 27(22):2079-2081

24. Park J, Tai YW, Cho D, Kweon IS (2017) A unified approach of multi-scale deep and hand-crafted features for defocus estimation. In: Proceedings of IEEE conference on computer vision and pattern recognition (CVPR). IEEE Computer Society Press, Los Alamitos, pp 1736-1745

25. Huang R, Feng W, Fan M, Wan L, Sun J (2018) Multiscale blur detection by learning discriminative deep features. Neurocomputing 285:154-166

26. Zhao W, Zhao F, Wang D, Lu H (2018) Defocus blur detection via multistream bottom-top-bottom fully convolutional network. In: Proceedings of IEEE conference on computer vision and pattern recognition (CVPR). IEEE Computer Society Press, Los Alamitos, pp 3080-3088
27. Zeng K, Wang Y, Mao J, Liu J, Peng W, Chen N (2019) A local metric for defocus blur detection based on CNN feature learning. IEEE Trans Image Process 28(5):2107-2115

28. Wang X, Zhang S, Liang X, Zhou H, Zheng J, Sun M (2019) Accurate and fast blur detection using a pyramid $\mathrm{m}$-shaped deep neural network. IEEE Access 99:86611-86624

29. Tang C, Zhu X, Liu X, Wang L, Zomaya A (2020) DeFusionNET: defocus blur detection via recurrently fusing and refining multi-scale deep features. In: Proceedings of IEEE conference on computer vision and pattern recognition (CVPR). IEEE Computer Society Press, Los Alamitos, pp 2695-2704

30. Zhao W, Zhao F, Wang D, Lu H (2020) Defocus blur detection via multi-stream bottom-top-bottom network. IEEE Trans Pattern Anal Mach Intell 42(8):1884-1897

31. Okassa AJO, Ngantcha JP, Allogho GG, Ele P, Okassa O (2020) Compression of vibration data by the Walsh-Hadamard transform. J Eng Appl Sci 15(10):2256-2260

32. Li J, Chen H, Duan X, Liu H, Ren Y (2019) An image invariant orthogonal moment based on a Walsh transform. J Harbin Eng Univ 10:1784-1789

33. Zheng P, Huang $\mathbf{J}$ (2018) Efficient encrypted images filtering and transform coding with Walsh-Hadamard transform and parallelization. IEEE Trans Image Process 99:1-1

34. Kanungo T, Mount DM, Netanyahu NS, Piatko CD, Silverman R, Wu AY (2002) An efficient k-means clustering algorithm: analysis and implementation. IEEE Trans Pattern Anal Mach Intell 24(7):881-892

35. Fino BJ, Algazi VR (2006) Unified matrix treatment of the fast Walsh-Hadamard transform. IEEE Trans Comput C-25(11):1142-1146

36. Larson EC, Chandler DM (2010) Most apparent distortion: fullreference image quality assessment and the role of strategy. $\mathrm{J}$ Electron Imaging 19:011006

37. Amer A, Dubois E (2005) Fast and reliable structure-oriented video noise estimation. IEEE Trans Circuits Syst Video Technol 15(1):113-118

38. Tang C, Liu X, Zheng X, Li W, Xiong W, Wang L, Zomaya A, Longo A (2020) DeFusionNET: defocus blur detection via recurrently fusing and refining discriminative multi-scale deep features. IEEE Trans Pattern Anal Mach Intell 99:1-1

39. Tang C, Liu X, Zhu X, Zhu E, Zomaya A (2020) R ${ }^{2}$ MRF: defocus blur detection via recurrently refining multi-scale residual features. Proc AAAI Conf Artif Intell 34(7):12063-12070

40. Li J, Fan D, Yang L, Gu S, Lu G, Xu Y, Zhang D (2021) Layeroutput guided complementary attention learning for image defocus blur detection. IEEE Trans Image Process 30:3748-3763

41. Dabov K, Foi A, Katkovnik V, Egiazarian K (2007) Image denoising by sparse 3-D transform-domain collaborative filtering. IEEE Trans Image Process 16(8):2080-2095

42. Mäkinen Y, Azzari L, Foi A (2020) Collaborative filtering of correlated noise: exact transform-domain variance for improved shrinkage and patch matching. IEEE Trans Image Process 29:8339-8354

Publisher's Note Springer Nature remains neutral with regard to jurisdictional claims in published maps and institutional affiliations. 